\title{
Role of Memory Formation (with Emphasis on Hippocampal Memory Formation) and Retrieval in Cognitive Learning
}

\author{
Basu $\mathrm{O}$ and Pandey SR \\ eWellness Expert, Kannamangala, Whitefield, Bangalore, India
}

*Corresponding author: Pandey SR Founder and CEO of eWellness Expert, Villa \# 39, Phase-2, MIMS Ardendale, Kannamangala, Whitefield, Bangalore, India, 560067, Tel: +918049545127, E-mail: shiva@ewellnessexpert.com

Citation: Basu O, Pandey SR (2017) Role of memory formation (with emphasis on Hippocampal memory formation) and retrieval in cognitive learning. J Neurosci Neuropsyc 1: 103. doi: 10.18875/2577-7890.1.103

Article history: Received: 15 June 2017, Accepted: 31 August 2017, Published: 01 September 2017

\begin{abstract}
We are virtually learning all the time from our sensory stimuli. Sometimes we do it consciously and sometimes unconsciously. Basically, we absorb certain information from outside, convert them to suit our physiology and process them to guide our behaviour and thoughts and that is the essence of learning. Cognitive science largely attributes this entire process to the brain, which is the main processing center for information, in a human body. Cognitive learning is associated with "thinking with the brain", to learn, however, renowned psychologists have confirmed that the human mind is not that developed to think. Thinking can be 'slow, effortful and uncertain', hence we have to rely on our past habits and memories to guide our actions, so that we do not have to think deliberately and deeply. This study aims to understand the role of memory formation in cognitive learning; we will also discuss the role of hippocampus in memory building and storing, based on established scientific theories, case studies and experiments. Retrieval of memories is also required to further cognitive learning, for if we cannot remember what we have learned, the stored the process previous information will be of no use; learning will not take place. In the paper, we will discuss about the effective ways in which stored memory can be retrieved easily, and help in learning.
\end{abstract}

Keywords: Memory formation; Hippocampal formation; Hippocampus; Cognitive brain research; Memory Retrieval

\section{Introduction}

Robert Sternberg, a cognitive scientist defines "Learning is any relatively permanent change in behaviour, thoughts or feelings of an organism that result from experience" [1,2]. Learning happens in different ways in different people. A child learns to sit, while crawling and then stands to walk; nobody ever teaches him to crawl, sit or stand on the feet; these learnings take place with natural urges and instincts to be self-dependent.

On the other hand, for the same child, reciting rhymes and songs come from learning with memory. Whatever, the child hears around, the words of his parents, noises, sound, music, television and mobile information, all stick to the mind easily and he is able to recollect the information very swiftly. Thus, learning happens very quickly, in a child, through active memory retrieval.

Learning can be of two kinds- implicit learning and explicit learning.

Implicit learning happens without our knowledge or awareness, for e.g. acquiring native language or mother tongue. It is similar to a child, learning to stand for the first time, or calling his mother's name.

Explicit learning is done with consciousness and purpose, for e.g. we go to school to learn English, Maths, Science, Geography etc. which are a part of our formal education, and we do it consciously through reading and memorizing, to develop our career.

Cognitive learning essentially speaks about 'thinking to learn', and it is stated that "memory is the residue of thought" However, Daniel Willingham says "people's minds is not especially well-suited to thinking. Thinking is slow, effortful and uncertain". This means we tend to automatically rely on past habits and memories to guide our actions and are less likely to think deliberately and deeply [3].

Hence the role of memory formation is possibly the most important factor for Cognitive Learning Theory [4]. 
Memory as defined by Merriam Webster is "the power or process of reproducing or recalling what has been learned and retained especially through associative mechanisms"; in other words, it is "the store of things learned and retained from an organism's activity or experience as evidenced by modification of structure or behaviour or by recall and recognition".

Cognitive science involves a variety of different research disciplines including psychology, computer science, linguistics, artificial intelligence, anthropology, philosophy and neuroscience, all striving to understand through interdisciplinary study, how human cognition and intelligence develops and shapes our behaviour. By studying human behaviour and performance, we can learn much about how memory works and how deep learning occurs.

\section{Multi-Store Model of Memory}

The multi-store model of memory, also known as modal model was proposed by Atkinson and Shiffrin in 1968, which stated that memory has mainly three storage components- namely, the sensory register or sensory memory, the short-term memory, and the long-term memory [5]. It is a structural model which helps understand the duration and amount of information stored in each component, thereby deducing the amount of learning that is taking place each time, an information is passed to our brain.

The sensory memory can store information for about $1 / 4^{\text {th }}$ to $1 / 2$ seconds, but it can receive huge amount of data or information from environmental stimuli. If we give attention to this information, it is passed to the short-term memory for temporary storage. Encoding is mainly sense specific in this component (Figure 1).

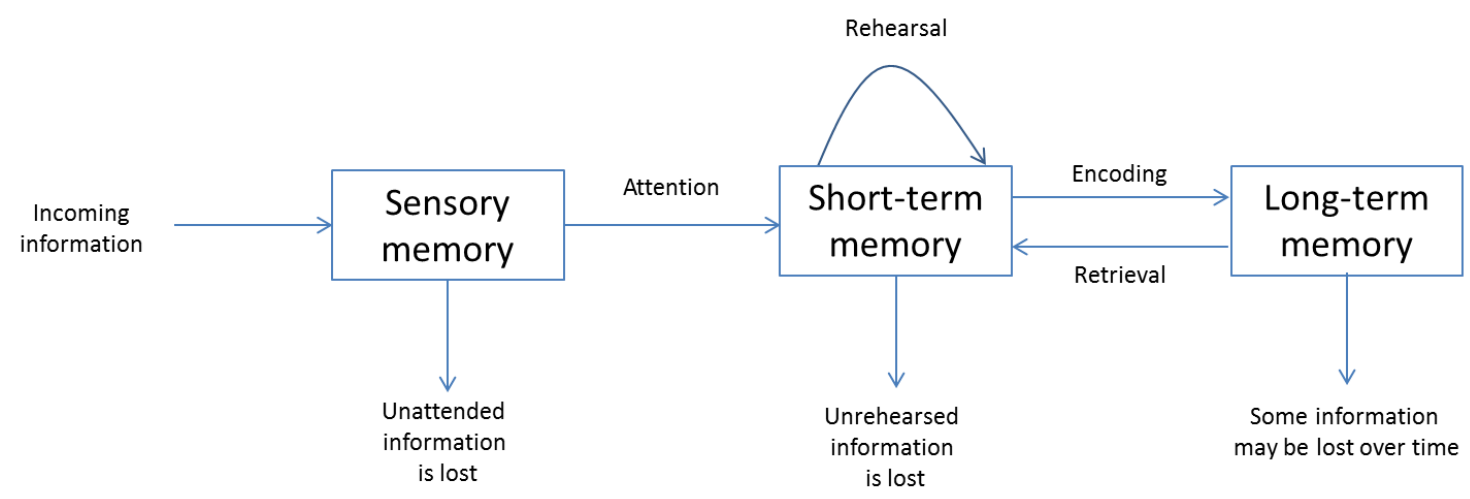

Figure 1: Structural features of memory and the control processes involved in forming memories [5]

The short-term memory can store limited amount of information for 5 to 30 seconds maximum; it can store $7(+$ or -2$)$ bits of information at a time. This component is sometimes treated as the working memory which holds information in the mind and manipulates it in the same way. Encoding happens through auditory means. Working memory is the gateway to long-term memory.

Long term memory has unlimited duration and capacity and encoding is mainly semantic though auditory and visual stimuli are entertained.

Learners can process only a limited amount of information at a time, hence, an active dialogue between working memory and long-term memory is required to form such that durable memories can be made in the process.

This is the essence of active learning. The dialogue between short-term memory and long- term memory make and store memories for new learning.

For e.g. in a classroom, a subject or chapter is taught in parts; each part scheduled for a particular period in a day. If a student merely sits and listens to the teacher, staring blankly, his learning will be slow, monotonous and often incomplete. On the other hand, a student who tries to associate what was taught in the previous class, on the same subject, while listening to the related new information on it, will develop active learning and better long-term memory. Many a times, learning happens through the process of recall. For e.g. we are in an airport for the first time; we will be seeing many new things and learn everything about flights and airways for the first time, directly; but suddenly, the word image Lufthansa on the digital display board reminds us of something we have seen earlier in films or tele-series; we immediately, can recall the information which was given regarding Lufthansa, in the context of the film or serial; when we try to associate the image with the past event, the previous information occurs parallelly, thus this constant retrieval and reorganization of thoughts help in better learning. We need to constantly navigate our already existing knowledge while making new memories. If knowledge is forgotten easily, learning is not complete.

Long-term memory is the most complex component of our memory system.

1. Creating long-lasting memories is most successful when new information is meaningfully linked to an already existing knowledge in our memories.

2. The more we process and think about something new, linking it to what we have learned earlier, the more enduring and retrievable the memories become. 
However, long-term memory is consolidated based on several non-cognitive factors as well that effect learning. Growth mindset, sense of belonging, academic perseverance as well as other personal qualities affects learning for a student.

There are factors which motivate the formation of long-term memory. For e.g. a person who is personally interested in animal anatomy, petting, dissection, nature colour etc. will learn biology quickly, will also be able to retrieve the memories better than others [6]. On the other hand, a student, who feels left out or less able in a classroom, will learn slowly, due to lack of belongingness in the platform of learning. If a learner believes that his or her ability and intelligence is limited, will face inhibitions in learning, that is the effect of a growth mindset.

\section{Review of Literature}

Neal J. Cohen and Jennifer Ryan, et al. (1999) concluded that the relational (declarative) memory account, in which it is proposed that the hippocampal system plays a critical role in binding together multiple inputs to permit representations of the relations among the constituent elements of scenes or events, can better accommodate the full range of imaging (and other existing) data than any other explanatory account of hippocampal function, using neuropsychological methods for determining the functional imaging work of the hippocampus.

R. J. Sutherland and J. W. Rudy (1989, Psychobiology) distinguished two major processes of memory formation, one involving a simple associative process, which does not depend on the hippocampal formation, and a configured associative process, which does. Results from six categories of research: place learning, recognition memory, latent inhibition, Pavlovian serial-compound dis- crimination learning, discrimination-reversal-leaming, and others were reviewed for reaching a conclusion. This work was supported in part by grants to R. J. Sutherland from the Natural Science and Engineering Research Council and Medical Research Council of Canada and to J. W. Rudy from the National Science Foundation (BNS 8207654). BA Van der Kolk discusses the role of neuroimaging in formation of traumatic memories and their retention, while LynnNadel and MorrisMoscovitch discusses the prime significance of damage to hippocampus, responsible for serious diseases like retrograde amnesia.

\section{Methodology}

Our aim is to understand the processes involved in memory formation and how the hippocampus of the brain helps in the processes of memory consolidation and forgetfulness [7]. Thus, we will analyse and understand the complex systems of the brain thereby determining their scope in learning. Interpreting the neurobiological processes of the brain is the method of approach to the study to keep the research simple and comprehensive.

\section{Role of Hippocampus in Memory Formation}

Historically, the link between the hippocampus and long-term memory formation was first described by William Scoville and Brenda Milner. The researchers reported about an individual who had undergone hippocampal surgery, to be relieved from epileptic seizures [8].

It was found, that after the surgery, the man was suffering from amnesia for a long time. He was unable to form new memories of everyday events, till a time was reached when he just had what scientifically is known as episodic memory [9]. The only memories he retained was that of his childhood.

Hippocampus is a small organ located within the brain's medial temporal lobe and forms an important part of the limbic system, the region that regulates emotions [10]. This organ is associated with memory, especially long-term memory formation, and also has important role in spatial navigation. Damage to this organ can lead to severe diseases of the brain like retrograde amnesia, Alzheimer's, confusion or delusion as because this is the first organ to be impaired after a brain injury (Figure 2).

Experts generally agree that the hippocampus plays a role in the formation of new memories and in the detection of new surroundings, occurrences and stimuli. Some also believe the organ is involved in declarative memory; that is memories that can be stated verbally such as facts and figures.

But most researchers do not believe that damage to hippocampus means memory loss, or inability to learn new things. In fact, experts have confirmed, that long-term memory depends on many other contributives of the brain, other than hippocampus and one who has a damaged hippocampus, is able to learn new skills like playing a musical instrument or solving puzzles.

Study on cellular processes of the brain have given evidences on potential role of metabotropic glutamate receptors, and more specifically the phospholipase C-coupled receptor 5, in long-term consolidation. The hyperexpression of receptor protein was limited to CA1 indicating a specific role of this brain region in the consolidation of memories.

But the role of hippocampus in developing spatial memory cannot be negated. Neuroscientists John O' Keefe and psychology professor Lynn Nadel studied the involvement of the hippocampus in memory formation and learning behaviours in the 1960's and 1970's. Together, they wrote the landmark 1978 book "The Hippocampus as a Cognitive map," which outlines the role of the hippocampus in learning and storing information referring to portions of space, in the form of cognitive maps [12]. 


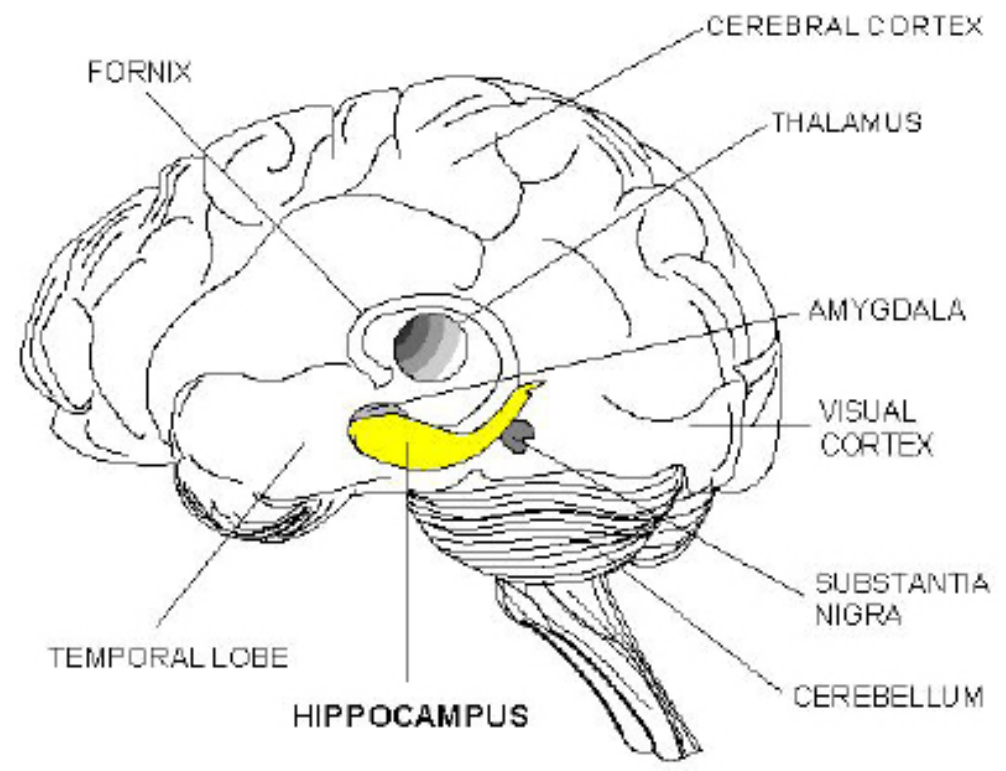

Figure 2: Brain areas associated with declarative memory disorders [11]

The process of consolidation of memory happens through a method called neurological potentiation. "Potentiation is the process by which synchronous firing of neurons makes those neurons more inclined to fire together in the future. Long-term potentiation occurs when the same group of neurons fire together so often that they become permanently sensitized to each other. As new experiences accumulate, the brain creates more and more connections and pathways, and may "re-wire" itself by re-routing connections and re-arranging its organization."

In this way, neural messages pass over and over again through an identifiable path of least resistance, and the flow is maintained by production of new proteins, building the synapses, responsible for consolidating or firmly holding the memories, without which the information get eroded or destroyed with time.

For e.g. if a stimulus like a word or music is heard or practiced again and again over the same scale, certain common synapses are built, which later on makes it easier for the brain to recall the notes without making mistakes. In this way, the brain organizes and reorganizes itself in response to identified stimuli, leading to formation of long term memory. The ability of the connection, or synapse, between two neurons to change in strength, and for lasting changes to occur in the efficiency of synaptic transmission, is known as synaptic plasticity or neural plasticity, and it is one of the important neurochemical foundations of memory and learning.

A single memory formation process can activate several groups of neurons in different part of the brain. The opposite of potentiation, is long-term depression, and can happen if neural messages over the networks are wrongly inhibited, by synaptic breakages.

The hippocampus is not involved with short-term memory and procedural memory types (memory of how to do motor actions, like walking). These are primarily handled by the cortex and the cerebellum.

But long-term depression can occur because of the building error of synapses of the cortex, the hippocampus, the striatum and other memory-related structures. Also, it can happen as a result of motor corrections (trying to correct procedural memory i.e. properly doing a task) in the cerebellum, located at the back of the brain.

Other experiments on animals have suggested that damage of hippocampus leads to hyperactivity, and disables a person from erasing previously learned information, when not required, thereby preventing formation of new memory which can be attentively stored for a long time. Delayed response tasks in monkeys, were studied to understand how Alzheimer's disease lead to erosion of memory in organisms [13]. Behavioural patterns in rats were studied to understand the learning method through memory formation and consolidation [14].

\section{Role of Memory Retrieval in Learning}

By now, we know that new learning should be securely consolidated and integrated into our long-term memory through active learning experiences.

But memories should also be readily accessible when required, so that we can recall what we have learned, when needed to produce. Retrieval is required everywhere.

In order to process new information better, we need to retrieve previous information in association to the new one, to make learning permanent.

We need to retrieve information for producing it in relevant context. 
We also need to retrieve memory in order to share the learning with another person.

\section{General hurdles in the path of retrieval}

Retrieval of memory is hard and deep enduring learning involves struggles, mistakes, confusion and requires persistent effort and engagement, especially for explicitly learning students. Lack of motivation can be a strong hurdle in the path of memory retrieval and active learning. If a person is never praised or acknowledged for his efforts, he will never repeat his action, never even try using his previous experiences in retrieval of memory, hence learning is curtailed.

Lack of proper cues can be a major drawback in case of retrieval of memory. For effecting active learning, the instructor must give prominent cues to the learner in the most strategic manner, which will help him think and process the previous learned information in association to the new information.

Multitasking can often lead to slow recall of memory, or easy forgetfulness. As we switch from one task to the other, new goals and rules intervene and they are as difficult as task varieties. For e.g. if a student is texting on his phone while learning a chapter from history book, and at the same time listening to the TV news, he is doing three tasks at a time, which involve different attention capacities, different goals and results, which make the complexity of tasks, greater.

\section{Ways for improving Retrieval of Memory}

Memories need to be readily accessible through recall cues that are triggered by circumstances, we want them to be used for.

Cued recall- which helps in the retrieval of memory happens with specific questions, which will trigger memories. For e.g. a student who was taught photosynthesis in biology class is given specific cues like "which gas is produced as a by-product of plants?", then memories of that specific information are triggered in the brain.

Free recall - hints or cues given are more generic in nature which influence retrieval of memories, e.g. if the same student is asked "what did you learn about photosynthesis today?"

Organization helps in retrieval. If a learner is given the opportunity to take information already put in memory and reorganize it in some way by thinking about connections and relationships; the more consolidated the memory subsequently becomes, easier it will be to retrieve later. Likewise, if the learner is made aware of organizational relationships during memory formation, the easier it will likely be to retrieve memories with more than one retrieval cue. Effective retrieval should take time. If personal meaning and purpose of learning is associated, retrieval happens in a better way.

Three things particular useful for students in improving cognitive learning are-

1. Retrieval Practice, which is recalling information to mind previously stored in long term memory overtime,

2. Spaced practice which means studying in shorter intervals and

3. Interleaving which means spacing practice while switching between different subjects.

Retrieval modifies and strengthens memories; creates strengthened or new retrieval pathways. It also activates and links related information, and reveals missing or inaccessible information.

\section{Results}

Hippocampus is a major organ in the temporal lobe of the brain, which actively participates in long term memory formation by building up the specific synapses as a response to repeated stimulus or experience. If this synaptic bond is interrupted in the hippocampus or striatum of the brain, long-term potentiation process is disrupted, causing erosion of memory. Loss of recall badly hampers learning since long-term memory is never consolidated. This generally happens if the hippocampus is damaged due to accident of tremendous psychic trauma. However, hindrance in the process of recall is also observed due to increased challenges like multitasking, disorganization, lack of motivation or lack of belongingness, weak attention span and faltered growth mindset.

Growth mind-set refers to a belief that our ability and intelligence are changeable; that high performance is due, mostly, to hard work and persistence. For e.g. "I can get better at this, if given some more time". This positive attitude towards learning is extremely important, besides neurobiological factors, in consolidation and retrieval of long-term memory.

\section{Conclusion}

Cognitive learning is a complete complex process focused on the brain; it works on the basis of memory formation which works on the Multi-store model of memory. The external stimulus enters the sensory memory, if unattended, then it exits the brain, if given sufficient priority and attention, then enters short-term memory, where it is stored for some time and then through encoding and retrieval enters the long-term memory, where it is stored for unlimited time. Memory helps in learning; active dialogue between working memory and long-term memory is the key to active learning. Retrieval or recall plays an important role in formation of long-term memory; the more we recall information in different context, the more consolidated it becomes. If we study the brain and its different areas, we need to focus on the hippocampus which is extremely important for new memory formation and its consolidation; if this area of brain is damaged, a person can suffer memory loss, Alzheimer's disease, and inhibition in retaining new memory for long. 


\section{References}

1. Riedel G, Micheau J (2001) Function of the hippocampus in memory formation: desperately seeking resolution. Prog Neuropsychopharmacol Biol Psychiatry 25: 835-53.

2. Steve N (2017) Definition of Learning by Robert Sternberg. Science of Learning. EdX Course Offers Teachable Moments. Integrated Learning Initiative.

3. Admin ERN (2017) “Why Don’t Students Like School? by Daniel Willingham, 2009." Educational Research Newsletter and Webinars.

4. Sarah MS (2013) Cognitive Learning Theory - Using Thinking to Learn. Psycology, Explorable.

5. Saul McLeod (2007) Multi Store Model of Memory. Simply Psychology.

6. Healthline Medical Team (2015) Hippocampus Function, Anatomy \& Definition. Body Maps. Healthline Media, United States.

7. Memory Consolidation (2017) The Human Memory.

8. Mandal A (2014) Hippocampus Functions. News Medical life sciences.

9. Burgess N, Maguire EA, O’Keefe J (2002) The human hippocampus and spatial and episodic memory. Neuron 35: 625-41.

10. Memory, Learning, and Emotion: the Hippocampus (2014) PsychEducation.org.

11. Beyondthedish (2015) Epilepsy Reduces The Formation of New Neurons in the Brain.

12. Jonides J, Lewis RL, Nee DE, Lustig CA, Berman MG, et al. (2008) The Mind and Brain of Short-Term Memory. Annual Review of Psych 59: $193-224$.

13. Rodriguez JS, Merle G Paule (2009) Working Memory Delayed Response Tasks in Monkeys. Methods of Behavior Analysis in Neuroscience. (2nd Edn) U.S. National Library of Medicine, United States.

14. Quillfeldt JA (2016) Behavioral Methods to Study Learning and Memory in Rats. Rodent Model as Tools in Ethical Biomedical Research. Springer $271-311$. 